\title{
LA ISLA DE CALOR URBANO DE MADRID DURANTE LA OLA DE CALOR DE JULIO 2015
}

\author{
Domingo F. RASILLA ÁLVAREZ ${ }^{1}$, Fernando ALLENDE ÁLVAREZ² \\ y Felipe FERNÁNDEZ GARCÍA ${ }^{2}$ \\ ${ }^{I}$ Depto. de Geografia, Urbanismo y OT, Universidad de Cantabria. \\ ${ }^{2}$ Depto. de Geografia, Universidad Autónoma de Madrid. \\ domingo.rasilla@unican.es, fernando.allende@uam.es, felipe.fernandez@uam.es
}

\section{RESUMEN}

Este trabajo analiza la isla de calor urbana de Madrid durante julio de 2015. Los registros históricos de temperatura confirman que siguió el patrón de anteriores eventos: número elevado de días muy cálidos, pero sin alcanzar records absolutos, y noches muy cálidas. El fenómeno de la isla de calor atmosférica estuvo presente casi todas las noches con una notable magnitud (entre $2-4^{\circ} \mathrm{C}$ ); por el contrario, durante las horas diurnas se registró una isla de frescor relativamente suave $\left(<1^{\circ} \mathrm{C}\right)$. Las imágenes de satélite revelan que el campo térmico superficial experimentó idéntico ciclo diario, estrechamente ligado a los usos del terreno, con la formación de una isla de calor nocturna de una magnitud similar a la atmosférica, y una isla de frescor diurna igual de potente. La correlación entre la evolución temporal de ambas manifestaciones, al igual que el contraste especial durante la noche más cálida, revela un comportamiento similar durante las horas nocturnas, lo cual ofrece perspectivas interesantes para la integración de ambas variables en estudios aplicados.

Palabras clave: Madrid, ola de calor, isla de calor urbano, Teledetección, MODIS.

\begin{abstract}
This paper analyzes Madrid's Urban Heat Island, comparing air and land surface temperature records during July 2015. The long-term analysis of temperature records from Retiro confirms a long spell of very warm days, without diurnal breaking records, but very hot nights. The atmospheric UHI was a permanent feature, reaching $2-4^{\circ} \mathrm{C}$ on average; by day, a "cool" island was also detected, but weaker. Satellite images reveal an identical daily cycle, closely related to land uses, the "diurnal cool island" becoming as intense as the nocturnal UHI. The temporal correlation among both UHI's in two selected locations, as well as its spatial structure during the warmest night shows good agreement, offering interesting perspectives for the integration of both variables in applied studies.
\end{abstract}

Key words: Madrid, Heat wave, Urban Heat island, remote sensing, MODIS

\section{INTRODUCCIÓN}

La isla de calor urbano, esto es, la diferencia de temperatura entre un núcleo urbano y sus alrededores, es un fenómeno común sobre múltiples localidades del planeta (Arnfield, 2003). El método de análisis tradicional ha sido la comparación entre los 
registros de la temperatura del aire de estaciones urbanas y rurales, o bien mediante transectos. Un método alternativo es la obtención de información térmica de la superficie terrestre, a través de sensores instalados en plataformas aerotransportadas. Éstos proporcionan información con mayor resolución espacial que las observaciones tradicionales. En comparación con otras plataformas (pe. ASTER o LANDSAT), su menor resolución espacial $\left(\sim 1 \mathrm{~km}^{2}\right)$ es compensada por una mayor resolución temporal (dos pasadas diarias), lo que permite estudiar la evolución del fenómeno tanto a escala anual como interanual, al disponerse de series de más de 15 años. Este producto ya ha sido usado en investigaciones sobre la UHI en ciudades de tamaños y climas diversos, por ejemplo en Europa (Pongracz et al., 2006; Cheval y Dumitrescu, 2009) y Asia (Hung et al., 2006).

Dado la creciente necesidad de adaptación a las consecuencias del calentamiento global en un contexto de envejecimiento de la población en el mundo desarrollado, y de crecimiento urbano explosivo en el resto, es necesario ofrecer un conocimiento lo más detallado posible de la dinámica espacio-temporal de este fenómeno para su incorporación en estudios sobre los riesgos en espacios urbanos (Rizwan et al., 2008). La vulnerabilidad de las áreas urbanas durante eventos extremos como las olas de calor ha quedado plenamente demostrada (Basu and Samet, 2002; Conti et al., 2005). El objetivo de esta aportación es investigar las características de la isla de calor de Madrid durante el mes de julio de 2015, en un contexto de ola de calor, comparando la isla de calor atmosférica con la superficial.

\section{DATOS Y METODOLOGÍA}

Los registros de temperatura del aire fueron proporcionados por la red sinóptica convencional de AEMET, utilizándose tanto las históricas (1861-2015) de temperatura diaria (máxima y mínima) como los valores diarios y horarios correspondientes a julio de 2015.

El análisis de la isla de calor superficial se ha llevado a cabo mediante el producto MYD11A1 (V5)-MODIS/Aqua Land Surface Temperature and Emissivity Daily L3 Global $1 \mathrm{~km}$ Grid SIN. Aunque el sensor MODIS (Moderate Resolution Imaging Spectroradiometer) está instalado en dos plataformas, Aqua y Terra, sólo se ha hecho uso de la primera, ya que su hora de paso (01:30 y 13:30) es más cercana a los momentos de máxima intensidad de la isla de calor urbana. Estudios previos han confirmado la precisión del producto MODIS LST (superior a $1{ }^{\circ} \mathrm{C}$ en el intervalo $-10 / 50^{\circ} \mathrm{C}$ ), particularmente bajo cielos despejados (Wan et al., 2004), aún mejor durante la noche (Rigo et al., 2006); el resto de detalles técnicos pueden ser consultados en la página web de la NASA (https://lpdaac.usgs.gov/). Las imágenes originales fueron sometidas a un análisis de control de calidad utilizando sólo aquellas con más del $90 \%$ de los pixeles válidos; las causas más habituales de eliminación son los errores del sensor y la presencia de nubes. Para relacionar los usos del suelo con la temperatura superficial se cruzaron las imágenes de satélite con la base de datos Global Land Cover 2000 (European Commission, Joint Research Centre, http:// forobs.jrc.ec.europa.eu/products/glc2000/glc2000.php). Ésta cubre todo el planeta, proporcionando 22 categorías con una resolución de $1 \mathrm{~km}^{2}$, obtenida a partir del sensor 
VEGETATION (satélite SPOT 4). Los tipos de uso de suelo se inspiran en los criterios proporcionados por la FAO (Land Cover Classification System, LCCS).

Para la definición de las “olas de calor”, se utilizó la metodología aplicada por AEMET, que propone que la temperatura máxima diaria supere el centil 95 de la serie histórica de los meses de julio y agosto (periodo 1971-2000) durante al menos tres días consecutivos

\section{CARACTERIZACIÓN TÉRMICA DE JULIO DE 2015}

En este apartado se analizan las temperaturas del citado periodo en el marco de la evolución térmica secular, como avance de las condiciones esperables en la ciudad de Madrid si se cumplen las previsiones de los modelos climáticos, y las causas sinópticas que propiciaron dicho episodio de calor.

\subsection{Caracterización térmica de julio 2015}

Julio de 2015 estuvo caracterizado por anomalías positivas de la temperatura en gran parte de Europa, con especial incidencia sobre la mitad oriental de la Península Ibérica, Italia y Balcanes. En lo que respecta a su comportamiento específico sobre España $\left(26,5^{\circ} \mathrm{C}, 2,5^{\circ} \mathrm{C}\right.$ por encima del periodo $\left.1981-2010\right)$ ha sido el mes más cálido nunca registrado (agosto de 2003 alcanzó 26,2 ${ }^{\circ} \mathrm{C}$; AEMET, 2015), aunque sólo un número reducido de localidades superó anteriores registros en las máximas absolutas. Los días más rigurosos fueron el 6 y el 7 de julio, cuando se alcanzaron valores por encima de $\operatorname{los} 45^{\circ} \mathrm{C}$ en el Guadalquivir y la mitad $\mathrm{E}$ de la península.

De acuerdo con el cuadro 1, el verano de 2015 se convirtió en el más cálido de todo el registro instrumental en el observatorio de Retiro, tanto en lo que hace referencia a las temperaturas máximas como a las mínimas. En comparación con 2003, las temperaturas máximas fueron $0,9^{\circ} \mathrm{C}$ más altas en 2015 , frente a $0,1^{\circ} \mathrm{C}$ en lo que hace referencia a las mínimas. Las mayores diferencias se observan, de hecho, al contrastar la variabilidad diaria: 2003 fue un verano caracterizado por una menor variabilidad térmica, tanto en las máximas (desviación típica de 20033,4 frente a $2015,4,3^{\circ} \mathrm{C}$ ) como en las mínimas $\left(2,7\right.$ frente a $\left.3,1^{\circ} \mathrm{C}\right)$.

\begin{tabular}{|l|c|c|c|c|}
\hline & $1861-2014$ & $1971-2010$ & 2015 & $\begin{array}{c}\text { Efeméride } \\
\text { anterior (yr) }\end{array}$ \\
\hline T media (JJA) & 22,9 & 23,8 & 26,5 & $26.2(2003)$ \\
\hline T media de las máximas (JJA) & 29,0 & 30,0 & 33,3 & $32.7(2009)$ \\
\hline T media de las mínimas (JJA) & 16,9 & 17,5 & 19,7 & $19.3(2003)$ \\
\hline T maxima absoluta (JJA) & 40,6 & 40,0 & 40,2 & $40,6(2012)$ \\
\hline T mínima absoluta (JJA) & 26,8 & 25,4 & 25,6 & $26,8(1903)$ \\
\hline
\end{tabular}

Tabla I: Valores térmicos más representativos del verano de 2015 con respecto a todo el periodo instrumental (1861-2014) y al periodo de referencia más reciente (1971-2000).

La notable variabilidad térmica del verano de 2015 se puede observar claramente en la evolución de las temperaturas máximas y mínimas del Retiro desde el 1 de junio hasta el 31 de agosto de 2015; la figura 1 también incluye sus diferencias respecto 
a los valores umbrales $\left(36,0^{\circ}\right.$ y $\left.22^{\circ} \mathrm{C}\right)$. El verano comenzó, paradójicamente, con un ambiente fresco, ya que casi todo el mes de junio registró temperaturas ligeramente por debajo de los valores normales. No obstante, en la segunda quincena de junio, un espectacular ascenso de las temperaturas llevó a una primera ola de calor entre los días 27 y 30 de junio, en los que se alcanzó una temperatura máxima de $39,6^{\circ} \mathrm{C}$. El periodo más prolongado y severo se extendió entre el 3 y el 17 de julio, alcanzándose una máxima de $40,2^{\circ} \mathrm{C}$ el 6 de julio (acompañado de una mínima la noche siguiente de $25,2^{\circ} \mathrm{C}$ ). Aunque en la segunda mitad de julio se produjeron 3 episodios más de calor, estuvieron separados por periodos más prolongados de amortiguación térmica.
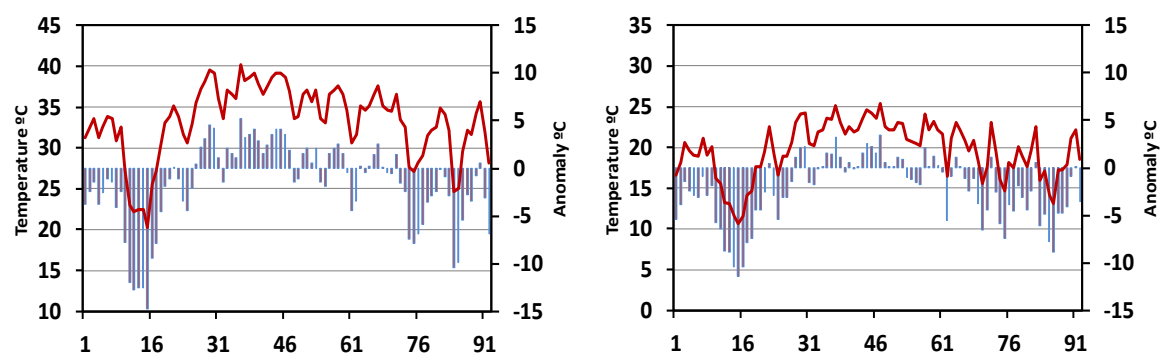

Figura 1: Evolución de las temperaturas máximas y mínimas desde el 1 de junio hasta el 31 de agosto de 2015 (línea) y anomalías diarias (barras) con respecto al periodo normal 1971-2010.

\subsection{Caracterización sinóptica}

Un rasgo compartido por las olas de calor en la Meseta Meridional es una circulación anticiclónica (figura 2); ésta favorece la advección de aire desde el $\mathrm{N}$ de África o el Atlántico subtropical; una subsidencia generalizada, que propicia un calentamiento adiabático adicional y valores elevados de radiación solar (García et al, 2002). El mapa de la topografía de $500 \mathrm{hPa}$ muestra una amplia dorsal, con un eje ligeramente inclinado de SSW a NNE. El mapa de la temperatura a $850 \mathrm{hPa}$, con anomalías superiores a $5^{\circ} \mathrm{C}$, muestra la potencia del evento.
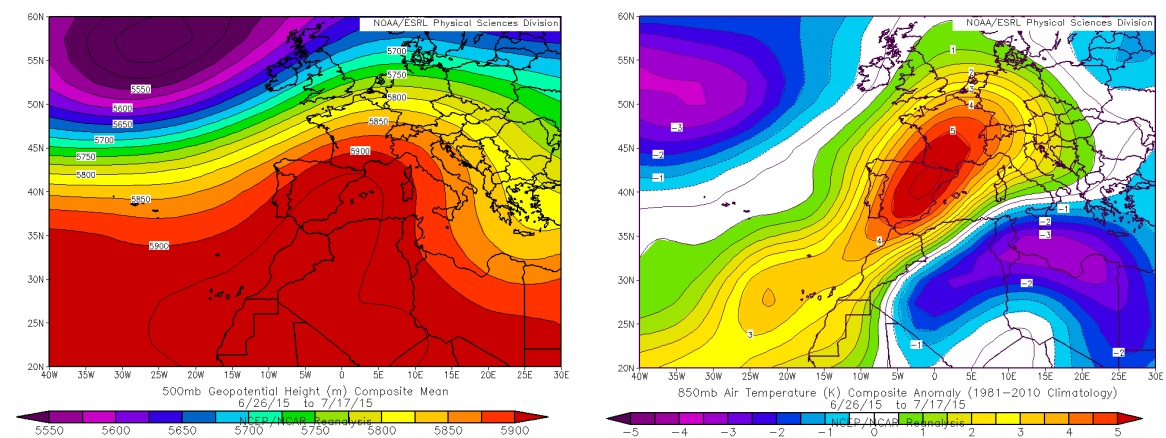

Figura 2: Topografia de la superficie de $500 \mathrm{hPa}$ y anomalia de la temperatura de $850 \mathrm{hPa}$ durante julio de 2015. Fuente: NOAA/ESRL Physical Sciences Division en http://www.esrl. noaa.gov/psd/. 


\section{LA ISLA DE CALOR URBANO DE MADRID EN JULIO 2015}

Esta apartado muestra los efectos de las altas temperaturas sobre la isla de calor urbana tanto atmosférica como superficial, y compara ambas.

\subsection{La isla de calor atmosférico}

Aunque la isla de calor urbano de Madrid es un fenómeno presente a lo largo de todo el año, su frecuencia e intensidad varía según las condiciones atmosféricas; las más favorables son elevada insolación y vientos en calma. A pesar de que en invierno se registran episodios muy intensos (enfriamiento de los ámbitos rurales durante inversiones térmicas), la frecuencia de islas de calor es más elevada en verano, como corresponde al predominio de esas condiciones favorables. Tomando como referencia Torrejón de Ardoz, la diferencia de las temperaturas nocturnas del el centro superó los $2,5^{\circ} \mathrm{C}$; por el contrario, durante el día, la situación se invirtió, con diferencias negativas próximas a $1^{\circ} \mathrm{C}$.

\begin{tabular}{|l|c|c|c|c|}
\hline & Tmax & Tmín & $\Delta$ diurna & $\Delta$ nocturna \\
\hline Torrejón & 37,4 & 20,1 & & \\
\hline Barajas & 37,1 & 20,7 & $-0,28$ & 0,61 \\
\hline Getafe & 36,8 & 23,0 & $-0,59$ & 2,84 \\
\hline Cuatro Vientos & 36,4 & 22,4 & $-0,92$ & 2,29 \\
\hline Retiro & 36,6 & 22,9 & $-0,82$ & 2,77 \\
\hline
\end{tabular}

Tabla II: Temperaturas máximas y mínimas en julio de 2015 en los observatorios de la red sinóptica de AEMET.

Una clasificación de su intensidad muestra que este fenómeno estuvo presente todas las noches de julio de 2015 , y en el $50 \%$ de los casos alcanzó una intensidad moderada o fuerte, siendo mayor en el centro (Retiro) que en la periferia (Cuatro Vientos). Además, mostró una relación inversa con la temperatura máxima ( $\mathrm{r}=0,60$ en Retiro y 0,58 en Cuatro Vientos); la baja correlación de Getafe $(0,28)$ hace pensar que sus altas temperaturas nocturnas son resultado de una localización meridional y de un terreno más favorable a la conservación del calor diurno.

\begin{tabular}{|l|c|c|}
\hline & Retiro & Cuatro Vientos \\
\hline Sin isla de calor & 0,0 & 3,2 \\
\hline Isla débil $\left(<2^{\circ} \mathrm{C}\right)$ & 6,5 & 16,1 \\
\hline Isla moderada $\left(2-4^{\circ} \mathrm{C}\right)$ & 54,8 & 51,6 \\
\hline Isla fuerte $\left(4-6^{\circ} \mathrm{C}\right)$ & 35,5 & 29,0 \\
\hline Isla muy fuerte $\left(>6^{\circ} \mathrm{C}\right)$ & 3,2 & 0,0 \\
\hline
\end{tabular}

Tabla III: Intensidad de la isla de calor atmosférica en Madrid en julio de 2015.

La isla de calor también sigue un ciclo diario, en el que las máximas diferencias ocurren poco antes del amanecer (figura 3). Las diferencias comienzan hacia las 22 horas, alcanzan su máximo a las 5 de la madrugada y desparecen a partir de las 8 horas, cuando la radiación directa eleva rápidamente las temperaturas en Barajas. A partir de las 13 horas la diferencia negativa entre Retiro y Barajas aumenta, proba- 
blemente por un enfriamiento ligado a la evapotranspiración del parque del Retiro. El régimen térmico de los restantes observatorios "no urbanos" ofrece singularidades que afectan a la cuantificación de la isla de calor. Pozuelo y Ciudad Universitaria, en los márgenes de la vaguada del Manzanares, muestran un perfil diurno idéntico, pero el mayor enfriamiento nocturno de Pozuelo $\left(4^{\circ} \mathrm{C}\right)$ resultaría de la topografía o a la distancia a Madrid, adelantándose dos horas a Madrid. A su vez, Ciudad Universitaria muestra una curva similar a Barajas durante la noche, pero sus valores se disparan por el día, debido a su mayor exposición a la radiación solar.

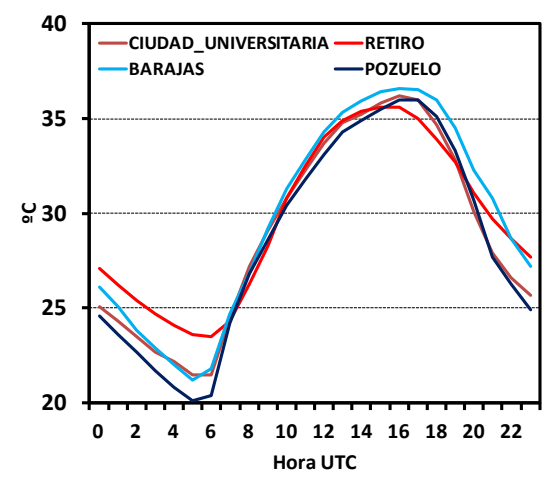

Figura 3: Ciclo horario de la temperatura durante julio de 2015. Fuente: elaboración propia a partir de datos de AeMet.

\subsection{Relación entre temperatura y usos de suelo: la isla de calor superficial}

La base de datos Global Land Cover 2000 considera el $14 \%$ del territorio analizado urbano; el $86 \%$ cultivos $(67,1 \%$, mayoritariamente de secano), arbolado el $9,06 \%$ y matorral/pastizal el 9,88\%. Los espacios urbanos se confirman como ámbitos con una gran inercia térmica y reducida amplitud diurna (Figura 4): son más frescos durante el día $\left(46,9^{\circ} \mathrm{C}\right)$ y más cálidos durante la noche $\left(23,1^{\circ} \mathrm{C}\right)$.
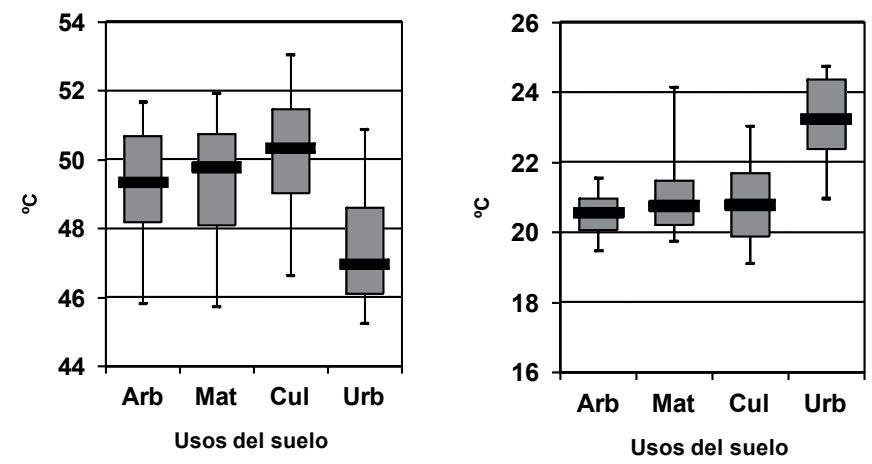

Figura 4: Distribución de la temperaturas de la superficie terrestre según usos de suelo durante julio de 2015 (derecha, imágenes nocturnas, izquierda, imágenes diurnas).

Fuente: elaboración propia a partir de datos del Global Land Cover 2000. 
La diversidad térmica es más elevada por el día en cualquier superficie (salvo la excepción de las superficies de matorral por la noche). Ello se explica por las múltiples reflexiones de la radiación solar asociada al recorrido del sol sobre el horizonte, las sombras generadas por los edificios o la presencia de masas de vegetación dentro de los ámbitos urbanos, frente a una emisión constante de radiación infrarroja durante la noche (Wang et al. 2008). Por otro lado, los usos urbanos muestran una distribución asimétrica de las temperaturas, con sesgo positivo (píxeles más cálidos) durante el día y negativo (píxeles más fríos) por la noche, mientras que los restantes ofrecen una distribución simétrica. La densidad de la cubierta vegetal es inversamente proporcional a la temperatura de la superficie durante el día, siendo los usos del suelo más frescos los correspondientes a las masas forestales. Por la noche, esta gradación se debilita, siendo característico el contraste en la reducida variabilidad térmica de las superficies arboladas y los máximos del matorral.

Los mapas correspondientes al promedio de la temperatura de la superficie muestran que tanto la isla de frescor diurno como la isla de calor nocturno coinciden en gran medida con el ámbito edificado, y particularmente con los barrios del centro de Madrid (figura 5). El mapa nocturno muestra una disposición concéntrica que contrasta con la disposición diurna, más irregular: durante el día, el volumen edificado, las sombras y la mayor capacidad calorífica de los materiales urbanos explica un lento calentamiento frente al resto de las superficies. Por la noche, el ritmo del enfriamiento se invierte por el calor acumulado en la ciudad. Además, por el día se aprecian anomalías cálidas sobre suelos desnudos (yesos) al SE de Madrid, al igual que el complejo aeroportuario de Barajas, mientras los píxeles más frescos se sitúan sobre superficies con disponibilidad de agua (regadíos del Jarama).
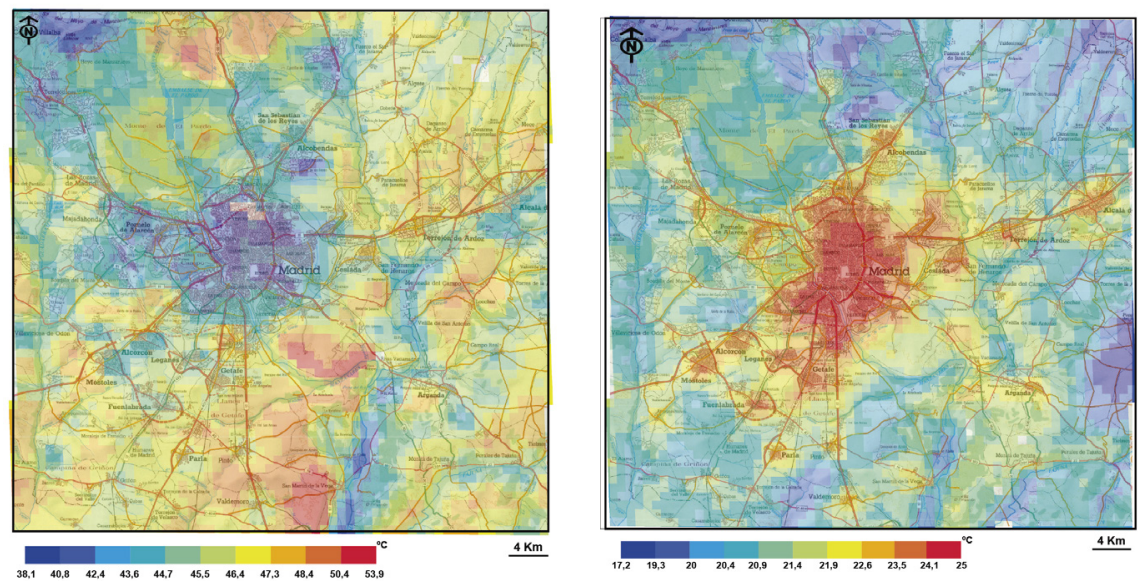

Figura 5: Patrones de la temperatura de superficie en julio de 2015 (derecha, imágenes nocturnas; izquierda, imágenes diurnas). Fuente: Elaboración propia a partir de datos MODIS MYD11 (Aqua).

Para determinar si existieron diferencias significativas en la intensidad y forma de las islas de calor y frescor durante julio de 2015 respecto a las observaciones entre 2002 y 2014, se elaboraron sendos transectos, uno N-S (izquierda) y otro W-E (dere- 
cha). De ellos se deduce que los valores registrados en 2015 sólo difieren del promedio a largo plazo en términos de magnitud, mientras que la forma de ambas islas no sufre excesivas modificaciones (figura 6).
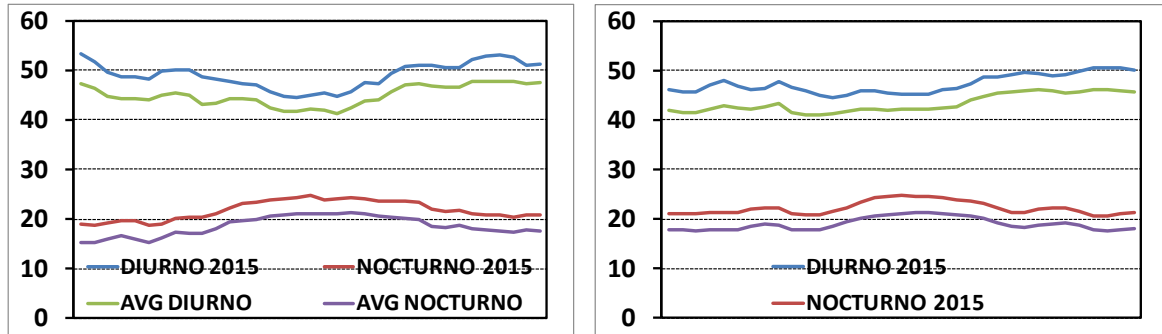

Figura 6: Transectos de la temperatura de superficie durante 2015 respecto al promedio 2002-2014 (derecha, transecto N-S, izquierda, transecto W-E).

\subsection{Comparación de los registros de MODIS LST con observaciones de temperatura del aire}

Con el fin de comprobar el grado de similitud entre las dos manifestaciones de la isla de calor, se comparó la evolución de la temperatura de superficie y la del aire en dos localizaciones representativas del comportamiento térmico de un ámbito rural (Barajas) y otro urbano (El Retiro). Los valores corresponden a la temperatura superficial del punto de rejilla más próximo a cada observatorio meteorológico (figura 7).
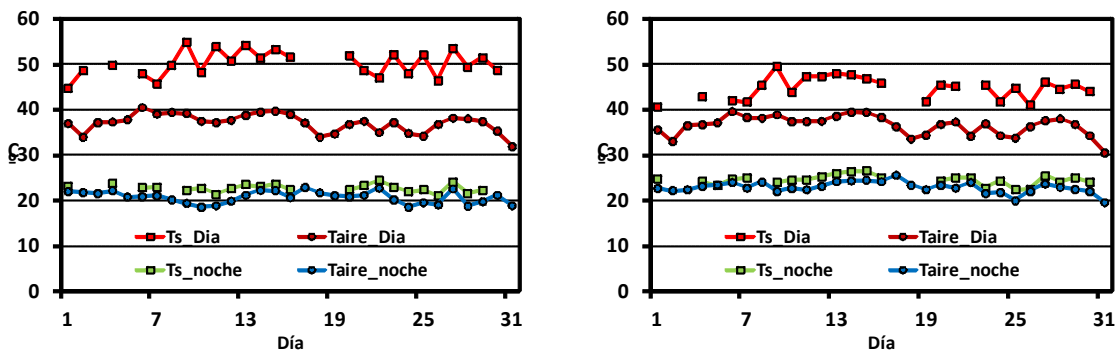

Figura 7: Evolución de las temperaturas de la superficie terrestre y del aire en Retiro (derecha) y Barajas (izquierda).

La magnitud de la temperatura de superficie difiere hasta $10^{\circ} \mathrm{C}$ de la temperatura del aire durante las horas diurnas en Barajas. Por el contrario, de noche, ambas curvas se solapan, si bien la temperatura de superficie se mantiene ligeramente más cálida. El coeficiente de correlación de Pearson confirma una variabilidad diaria similar en el caso de las mínimas (Barajas 0,85 frente a 0,79 en Retiro), pero mucho peor durante el día sobre superficies no urbanas (Barajas 0,26 frente a 0,49 en Retiro). La relación entre la temperatura del aire y la de superficie durante la noche también es reseñable a escala espacial. Durante la noche más cálida (madrugada del 7 de julio), el valor del coeficiente de determinación demuestra que ambas magnitudes comparten más del $50 \%$ de la variabilidad, respondiendo a mecanismos similares (usos de suelo). 
Téngase en cuenta que no son magnitudes equivalentes, ya que se comparan la temperatura mínima del aire en una serie de localizaciones puntuales y la temperatura de la superficie en los píxeles correspondientes a su emplazamiento, y que la red de observatorios está a cargo de "no profesionales".

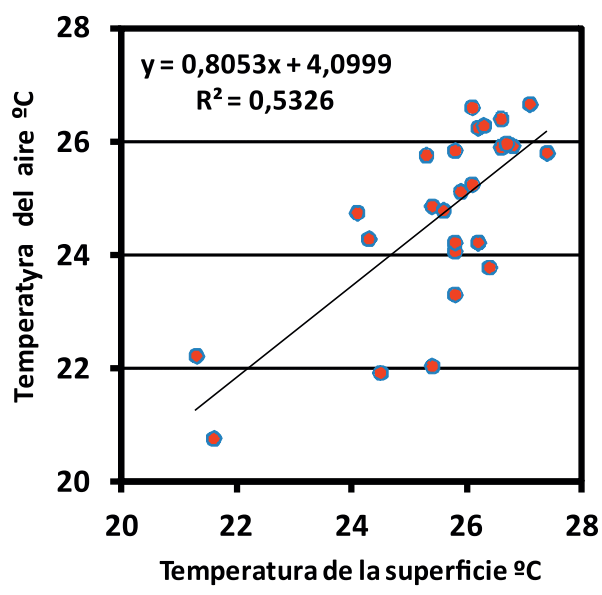

Figura 8: Correlación entre la temperatura del aire (red METEOCLIMATIC, http://www.meteoclimatic.net/) y la temperatura de la superficie terrestre en Madrid durante la noche del 6-7 de julio de 2015.

\section{CONCLUSIONES}

Este trabajo analiza la isla de calor urbana de Madrid durante julio de 2015, que podría considerarse un buen análogo de las condiciones estivales en los próximos decenios, combinando información procedente de estaciones meteorológicas convencionales e imágenes de satélite (sensor MODIS).

La isla de calor atmosférica estuvo presente casi todas las noches, con notable magnitud; por el contrario, durante las horas diurnas se registró una isla de frescor relativamente suave. Las imágenes de satélite revela una isla de calor nocturna, de una magnitud similar a la atmosférica, y una isla de frescor diurna tan potente como la nocturna. La correlación temporal entre ambas manifestaciones durante todo el mes, al igual que la correlación espacial durante la jornada de temperaturas más altas revela un comportamiento similar durante las horas nocturnas.

Un resultado sorprendente es la imposibilidad de confirmar una intensificación de la isla de calor nocturna superficial durante episodios cálidos. Una posible explicación es el uso de imágenes de satélite correspondientes a noches con las mejores condiciones para la formación de la isla de calor, a diferencia todas las noches disponibles para la isla de calor atmosférica (éstas incluyen situaciones inestables que limitan la intensidad de la UHI nocturna). Otra posibilidad es el desfase horario entre las temperaturas mínimas (la máxima intensidad de la isla atmosférica ocurre poco antes del amanecer), frente a la observación del satélite, aproximadamente a las 01:30 de la noche. 


\section{REFERENCIAS}

AEMET (2015), Informe Mensual Climatológico Febrero 2015. [http://www.aemet. es/documentos/es/serviciosclimaticos/vigilancia_clima/resumenes_climat/ mensuales/2015/res_mens_clim_2015_07.pdf]

Arnfield A.J. (2003). Two decades of urban climate research: a review of turbulence, exchanges of energy and water, and the urban heat island. International Journal of Climatology 23: 1-26, doi:10.1002/joc.859.

Basu, R. y Samet, J.M. (2002). Relation between elevated ambient temperature and mortality: a review of the epidemiologic evidence. Epidemiologic Reviews 24: 190-202, doi:10.1093/epirev/mxf007.

Cheval, S.; Dumitrescu, A. y Bell, A. (2009). The urban heat island of Bucharest during the extreme high temperatures of July 2007. Theoretical and Applied Climatology 97: 391-401, doi:10.1007/s00704-008-0088-3.

Conti, S.; Meli, P.; Minelli, G.; Solimini, R.; Toccaceli, V.; Vichi, M.; Beltrano, C. y Perini, L. (2005). Epidemiologic study of mortality during the Summer 2003 heat wave in Italy. Environmental Research 98: 390-399, doi:10.1016/j. envres.2004.10.009.

García-Herrera, R.; Prieto, L.; Díaz, J.; Hernández, E. y del Teso, T. (2002). Synoptic conditions leading to extremely high temperatures in Madrid. Annales Geophysicae 20: 237-245.

Hung, T.; Uchihama, D.; Ochi, S. y Yasuoka, Y.(2006). Assessment with satellite data of the urban heat island effects in Asian mega cities. International Journal of Applied Earth Observation and Geoinformation 8: 34-48, doi:10.1016/j. jag.2005.05.003.

Karl, T.; Diaz, H. y Kukla, G. (1988). Urbanization: Its detection and effect in the United States climate record. Journal of Climate 1: 1099-1123, doi:10.1175/15200442(1988)001<1099: UIDAEI>2.0.CO;2.

Kukla, G.; Gavin, J. y Karl, T. (1986). Urban warming. Journal of Applied Meteorology 25: 1265-1270, doi:10.1175/1520-0450(1986) 025<1265:UW>2.0.CO;2.

Pongracz, R.; Bartholy, J. y Dezso, Z. (2006). Remotely sensed thermal information applied to urban climate analysis. Advances in Space Research 37: 2191-2196, doi:10.1016/j.asr.2005.06.069.

Rigo, G.; Parlow, E. y Oesch, D. (2006). Validation of satellite observed thermal emission with in-situ measurements over an urban surface. Remote Sensing of Environment 104: 201-210.

Rizwan, A.; Dennis, L. y Liu, C. (2008). A review on the generation, determination and mitigation of Urban Heat Island. Journal of Environmental Sciences 20: 120 128, doi:10.1016/S1001-0742(08)60019-4.

Smith, C.; Lindley, S. y Levermore, G. (2009). Estimating spatial and temporal patterns of urban anthropogenic heat fluxes for UK cities: the case of Manchester. Theoretical and Applied Climatology 98: 19-35, doi:10.1007/s00704-008-0086-5.

Wan, Z. (2008). New refinements and validation of the MODIS Land-Surface Temperature/Emissivity products. Remote Sensing of Environment 112: 59-74.

Wan, Z., Zhang, Y., Zhang, Y. Q., \& Li, Z. -L. (2004). Quality assessment and validation of the global land surface temperature. International Journal of Remote Sensing, $25,261-274$. 